\title{
Wasch' mir den Pelz, aber mach mich nicht nass!
}

\section{Die Kritik am EGMR hält wissenschaftlicher Betrachtung nicht stand}

\author{
Ludwig A. Minelli*
}

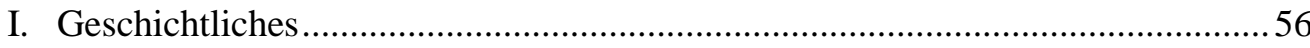

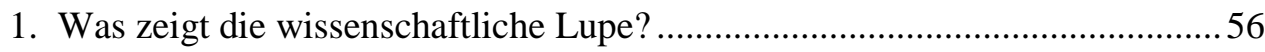

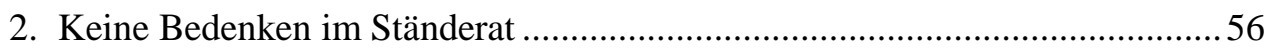

3. Von fremden Richtern kann nicht die Rede sein.............................................57

4. Präventive Wirkung der Konvention ...........................................................57

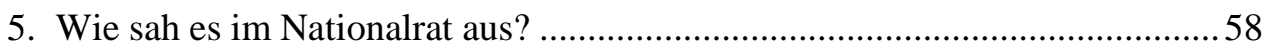

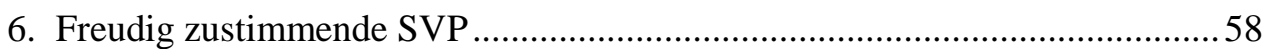

7. Bedauern wegen fehlender Ratifikation zweier Zusatzprotokolle.....................58

8. Relativierte Bedenken von James Schwarzenbach........................................59

II. Die «Bilanz» der Beschwerden gegen die Schweiz ..............................................60

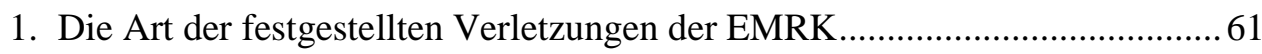

2. Unserem Rechtsempfinden widerstrebende Urteile? .....................................61

3. Zehn Fälle in Ausweisungssachen - nur drei Verurteilungen ..........................62

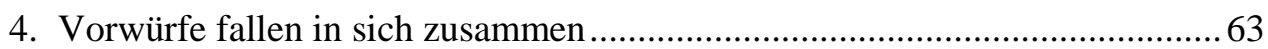

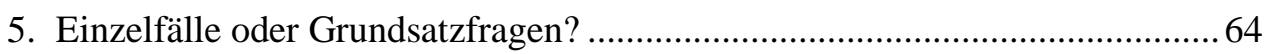

6. Der Anspruch auf einen sicheren Suizid auch ohne Krankheit .......................64

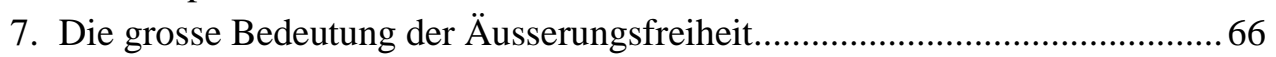

8. Vereinigungsfreiheit als wichtiges politisches Recht ...................................66

9. Heikle Abgrenzungen im Recht können zu Diskriminierung führen ...............67

III.Fazit

Zitiervorschlag: Ludwig A. Minelli, Wasch' mir den Pelz, aber mach mich nicht nass!, in: sui-generis 2014, S. 55

URL: $\quad$ sui-generis.ch/5

DOI: $\quad$ https://doi.org/10.21257/sg.5

Ludwig A. Minelli, geb. 1932, früher Journalist für «Die Tat», «Blick», «United Press International», zahlreiche Schweizer Tageszeitungen und erster Schweizer Korrespondent des deutschen Nachrichtenmagazins «Der Spiegel» (19641974), hat erst mit 44 Jahren das Studium der Rechte aufgenommen - angestossen durch einen Vortrag von Prof. Jörg Paul Müller im Zürcher Juristenverein über «Die EMRK und die Schweiz» am 29. November 1973. 1981 erwarb er an der Universität Zürich seinen lic.iur., mit 54 Jahren das Zürcher Rechtsanwaltspatent. 1978 gründete er die «Schweizerische Gesellschaft für die Europäische Menschenrechtskonvention (SGEMKO)» und 1998 den Verein «Dignitas - Menschenwürdig leben - Menschenwürdig sterben». Schon vor seinem Jurastudium wirkte er für die res publica; so verhalf er 1960 der Schulgeldfreiheit an Mittelschulen zum Durchbruch und verhinderte 1997 deren Wiedereinführung. 1965 sorgte er für die Wie- derentdeckung des Instruments der parlamentarischen Initiative im Bund. Generationen von Juristen lernten die Urteile des Bundesgerichtes in ihrer Studienzeit kennen, die seinen Namen tragen und die Praxis der abstrakten Normenkontrolle kantonaler Erlasse initiierten, die er in den Jahren 1973-1992, somit teilweise noch als Nicht-Jurist, erstritten hatte. Minelli führte zahlreiche EMRK-Verfahren gegen die Schweiz in Strassburg und lebt auf der Forch bei Zürich. Interessenbindungen des Autors: Der Autor ist Gründer und Generalsekretär der Schweizerischen Gesellschaft für die Europäische Menschenrechtskonvention (SGEMKO) (1978), Gründer und Generalsekretär des Vereins Dignitas - Menschenwürdig leben - Menschenwürdig sterben (1998). Er hat gemeinsam mit dem 2003 verstorbenen Zürcher Anwalt Manfred Kuhn seinerzeit Herbert Eggs und später Peter Herzig (im Verfahren Santschi und andere gegen die Schweiz) vor der Kommission vertreten. 
In der «Neuen Zürcher Zeitung» vom 26. Juni $2014^{1}$ hat sich der ehemalige Botschafter der Schweiz beim Europarat in Strassburg (20072011), Paul Widmer, in der Debatte über die Urteile des Europäischen Gerichtshofes für Menschenrechte (EGMR) in Schweizer Fällen $z u$ Wort gemeldet und in das bereits bekannte Horn «Mehr richterliche Zurückhaltung in Strassburg» gestossen. Er hat sich damit in die Reihe jener gestellt, die seit längerem den Versuch unternehmen, den EGMR gegenüber der Bevölkerung mit ähnlichen oder gleichlautenden und meist populistischen Argumenten zu diskreditieren ${ }^{2}$.

\section{Geschichtliches}

\section{Was zeigt die wissenschaftliche Lupe?}

1 Am 28. November 2014 kann die Schweiz die 40jährige Zugehörigkeit wenigstens zu einem Teil der Europäischen Menschenrechtskonvention (EMRK) feiern ${ }^{3}$. Da erscheint es als angebracht, Widmers Überlegungen unter die wissenschaftliche Lupe zu nehmen und deren Gehalt anhand der Fakten zu überprüfen.
2 Widmer hält zutreffend fest, dass die Schweiz erst 1963 dem Europarat beigetreten ist und sich viel Zeit gelassen hat, bis sie 1974 die EMRK ratifizierte ${ }^{4}$. Die Vorbehalte im Parlament seien gross gewesen, doch habe der damalige Vorsteher des Politischen Departements - wie das heutige Eidgenössische Departement des Äusseren (EDA) vormals hiess -, die Volksvertreter zu beschwichtigen gewusst.

\section{Keine Bedenken im Ständerat}

3 Der Glarner Ständerat Peter Hefti (FDP) wies in seinem Referat als Kommissionspräsident bezüglich der Ansätze zu Menschenrechten weit in die Antike zurück, indem er den Gegensatz zwischen den freiheitlichen alten Griechen und den tyrannischen Herrschern des antiken Persiens aus der Geschichtsschreibung Herodots erwähnte 5 . Er sprach auch davon, dass es darauf ankomme,

"auf welche Weise die Menschenrechte in der Praxis angewendet werden. Ihr Gehalt, ihre Tragweite, ihr Erfolg oder ihr Misserfolg, ihr letzten Endes wohltuender
Dieses Werk ist lizenziert unter einer Creative Commons Namensnennung - Weitergabe unter gleichen Bedingungen 4.0 International Lizenz.

haltung in Strassburg).

2 So etwa alt Bundesgerichtspräsident Martin Schubarth, Im kleinen Kreis der Erlauchten, Weltwoche Nr. 48/2012, Bundesrichterin Brigitte Pfiffner und Bundesgerichtsschreiberin Susanne Bollinger, Ausweitung konventionsgeschützter Rechte durch den EGMR und Probleme der innerstaatlichen Umsetzung, Jusletter 21. November 2011, sowie eine Reihe von Notabeln aus der SVP; humanrights.ch, Politische Folgen der SVPPolemik gegen den Europäischen Gerichtshof für Menschenrechte (Stand: 12.07.2014).

3 Die Schweiz hat bis heute das 1. Zusatzprotokoll zur EMRK trotz Unterzeichnung nicht ratifiziert, und zum Protokoll Nr. 4 zur EMRK stehen sowohl Unterschrift als auch Ratifizierung seitens der Schweiz aus. Dies, obwohl seit langem Staaten in Europa dem Europarat und damit der EMRK nur noch beitreten können, wenn sie den gesamten Kodex dieses einzigartigen Instruments zum Schutze der Menschenrechte und Grundfreiheiten als Ganzes übernehmen. Die Schweiz befindet sich damit bezüglich des Zusatzprotokolls noch in der Gesellschaft des Zwergstaats Monaco, und in Bezug auf Protokoll Nr. 4 auf einer Linie mit Griechenland; Grossbritannien und die Türkei haben zwar unterzeichnet, das Protokoll Nr. 4 aber bislang nicht ratifiziert.

4 Bundesrat Pierre Graber hinterlegte die schweizerische Ratifikationsurkunde am 28. November 1974 in Strassburg persönlich, nachdem der Ständerat dem Beitritt zur EMRK am 27. Juni 1974 (Amtl. Bull SR 1974,378390) einstimmig mit 27 Stimmen und der Nationalrat am 3. Oktober 1974 mit 87 gegen 15 Stimmen (Amtl. Bull NR 1974, 1461-1473 und 1502-1504) zugestimmt hatten, was gar auf einer Gedenktafel im Gebäude des Europarates in Strassburg dokumentiert worden ist, als ob es sich dabei um ein europäisches Wunder gehandelt hätte.

5 Amtl.Bull. SR 1974, 378. 
oder denken wir an die Weimarer Republik - schädigender Einfluss ergibt sich erst aus der Praxis des Behörden, welche über die Anwendung entscheiden. Bei keinen anderen Rechtsnormen trifft das auch nur entfernt so zu wie hier. Dass diesen Behörden ein weiter Spielraum verbleibt, ist allgemein anerkannt und hat die Geschichte immer wieder gezeigt. Ich zitiere aus einem Artikel in der ,Neuen Zürcher Zeitung " von Professor Wildhaber, der sich seinerzeit auf Professor Hans Huber berief: „Die Konkretisierung der Grundrechte durch den Verfassungsrichter ist eine spezifisch rechtsschöpferische, über die normale Auslegung hinausgehende Aufgabe, mehr Sinngebung als Sinndeutung. " ${ }^{6}$

\section{Von fremden Richtern kann nicht die Rede sein}

4 Raymond Broger (AI, CVP) betonte in seinem Votum,
«(d)ie in dieser vorliegenden Konvention geschützten Menschenrechte sind im Grunde genommen durchaus selbstver- ständlich. Wir bewegen uns damit inner- halb der westeuropäischen Rechtskultur, und es fällt uns kein Stein aus der Krone, wenn wir dies durch die Ratifikation auch ausdrücklich anerkennen. Im vorliegen- den Fall kann von einem fremden Richter überhaupt nicht die Rede sein.» ${ }^{7}$

5 Mathias Eggenberger (SG, SP) begrüsste die Vorschläge des Bundesrates vorbehaltlos.

«Es ist schon durch Herrn Broger darauf hingewiesen worden, dass doch ein ganz. wesentlicher Unterschied besteht zwischen dem, was die alten Eidgenossen als fremde Richter bezeichnet und was sie abgelehnt haben, und dem, was wir jetzt $z u$ tun im Begriffe sind. Jene fremden Richter sollten uns von aussen, von einer fremden Rechtsauffassung her, aufgezwungen werden; wir entscheiden völlig autonom und frei über den Beitritt zur Konvention und damit auch zur Anerkennung des europäischen Gerichtshofes. ${ }^{8}$

\section{Präventive Wirkung der Konvention}

6 Ferruccio Bolla (TI, FPD) fragte den Ständerat, was ein wirkliches oder vermeintliches Opfer eines Missbrauchs oder einer willkürlichen Entscheidung im Bereich der von der EMRK geschützten Bereiche wohl denke: ob es durch die inländische Rechtsprechung oder aber durch den internationalen Schutz besser gestellt werde? Er wies dann auf die bisherige Statistik der damaligen Europäischen Menschenrechtskommission hin, wonach nur gerade zwei Prozent der eingereichten Beschwerden überhaupt geprüft werden. Die Wirkung der EMRK erfolge weniger durch die auf ihr beruhenden Urteile, denn auf dem Einfluss, welchen sie durch ihre blosse Existenz auf die Handhabung des Rechts in den Mitgliedstaaten ausübe ${ }^{9}$.

7 Franz-Xaver Leu (LU, CVP) hielt fest:

«Es war deshalb eine der vornehmsten, wichtigsten und wesentlichsten Aufgaben des Europarates, diese Menschenrechte in einer Charta zusammenzufassen. Und dass das, was wir in unserem Lande hochhalten und was in unseren Verfassungen des Bundes und der Kantone niedergelegt ist, in einer europäischen Charta zugrunde gelegt wird, das kann uns nur mit grosser Genugtuung erfüllen. Unser Beitritt ist ein Akt der Solidarität mit den freiheitlich und demokratisch
Siehe Fussnote 5.

Amtl.Bull. SR 1974, 381.
Amtl.Bull. SR 1974, 382.

Amtl.Bull. SR 1974, 383. 
gesinnten Staaten Europas, und damit unterscheiden wir uns in ganz wesentlichsten und entscheidenden Punkten von den kommunistischen Staaten, die diese Menschenrechte nicht kennen. Wir müssen auch daran denken, dass gerade durch diese Menschenrechte die Grundlage geschaffen wird zur Verständigung der Völker untereinander.» ${ }^{10}$

So konnte sich Bundesrat Pierre Graber am Schluss der Debatte im «Stöckli» über den einhellig zum Ausdruck gebrachten Willen freuen, die Menschenrechte in der Schweiz durch den Beitritt zur EMRK zu stärken ${ }^{11}$.

\section{Wie sah es im Nationalrat aus?}

9 Im Ständerat ist somit von grossen Vorbehalten gar nichts zu finden. Wie sah es im Nationalrat aus?

Kommissionspräsident Walter Renschler (ZH, SP) bemerkte in durchaus zutreffender Weise, die Schweiz sei jenes Land, welches als

«einziges und letztes Mitglied des Europarates» der EMRK noch nicht angehöre; europäisch betrachtet sei somit «der Beitritt der Schweiz zur Menschenrechtskonvention nicht als mutiges Vorrücken, sondern eher als notwendiges Nachrücken zu bewerten» ${ }^{12}$.

11 Pier-Felice Barchi (TI, FDP), zweiter Berichterstatter, befasste sich vorwiegend mit dem Rang der EMRK im Verhältnis zur Bundesverfassung und $\mathrm{zu}$ Bundesgesetzen und hielt fest, solange der Gesetzgeber beim Erlass eines neuen Bundesgesetzes selbst nicht kundtue, dass er darin eine Bestimmung in offenem Widerspruch zur EMRK einfüge, sei davon auszugehen, dass jedes Bundesgesetz, auch wenn es nach Beitritt zur EMRK geschaffen worden sei, im Lichte der EMRK ausgelegt werden müsse ${ }^{13}$.

\section{Freudig zustimmende SVP}

12 Elisabeth Lardelli (GR, SVP) beantragte namens ihrer Fraktion Zustimmung zum Beitritt zur EMRK:

«Die Fraktion ist der Auffassung, dass die Menschenrechtskonvention, die aus der Konfrontation der westlichen Demokratien mit dem kommunistischen System entstanden ist, ein wirksameres Mittel darstellt, um dem Gedanken an ein geeintes Europa zu dienen... Unsere Fraktion betont auch den Gedanken, dass wir durch die Ratifizierung der Konvention ein Bollwerk gegenüber den Staaten setzen sollen, die die Menschenrechte mit Füssen treten.» ${ }^{14}$

13 Gabrielle Nanchen (VS, SP) begrüsste namens ihrer Fraktion, dass die Schweiz der EMRK endlich beitrete; dieses Instrument entspreche zwei Prinzipien der Sozialdemokraten: erstens die Bestätigung und Garantie der individuellen Freiheiten, und zweitens die Verstärkung internationaler Solidarität. Die schönsten Prinzipien gälten jedoch lediglich in dem Umfange, in welchem sie durch die Tatsachen gelebt werden ${ }^{15}$.

\section{Bedauern wegen fehlender Ratifikation zweier Zusatzprotokolle}

14 Der Fraktionssprecher des Landesrings der Unabhängigen, Claudius Alder (BL), meinte, die

«heute zur Debatte stehende Genehmi-
10 Amtl.Bull. SR 1974, 385.

Amtl.Bull. SR 1974., 385-387.

12 Amtl.Bull. NR 1974, 1462.
Amtl.Bull. NR 1974, 1465.

Amtl.Bull. NR 1974, 1467.

Siehe Fussnote 14. 
gung der Europäischen Menschenrechtskonvention bildet eine reichlich späte Einlösung eines Versprechens, des Versprechens des Bundesrates nämlich, dieses bedeutendste Vertragswerk des Europarates zu ratifizieren.»

15 Er brachte aber auch seine Enttäuschung zum Ausdruck, dass das Zusatzprotokoll und das Protokoll Nr. 4 nicht gleichzeitig ratifiziert werden ${ }^{16}$.

Carlo Speziali (TI, FDP) hielt fest, es sei überraschend, wahrzunehmen, dass die Länder, in welchen sich die grundlegende Philosophie und Moral der Menschenrechte entwickelt habe, nämlich Frankeich und die Schweiz, als letzte Staaten der EMRK beitreten. Der Bundesrat habe angesichts zahlreicher Einschränkungen lange gezögert. Auch er bedauerte, dass das Zusatzprotokoll nicht ratifiziert werden soll ${ }^{17}$.

17 Elisabeth Blunschy (SZ, CVP) äusserte sich dahingehend, es gehe darum, «dass wir die Menschenrechte in unserem eigenen Land anerkennen, als rechtlich durchsetzbar erklären und darüber hinaus mithelfen, dass diesen Rechten auch völkerrechtlich Nachachtung verschafft werde» $^{18}$.

\section{Relativierte Bedenken von James Schwarzenbach}

18 James Schwarzenbach (ZH, Republikaner), war der Einzige, der gegen eine Ratifikation sprach:

«Meine Bedenken, die gegen die Ratifikation der Menschenrechtskonvention vorzubringen sind, liegen demnach nicht in der Erklärung an sich, sondern in der neuerlichen Beschneidung unserer garantierten Souveränität, indem in Zukunft die schweizerische Legislative zu einer die Wünsche des Auslandes berücksichtigenden nachvollziehenden Behörde wird und indem das Recht auf Individualbeschwerde in Strassburg dem Urbegehren der Bundesgründer, keine fremden Richter zu dulden, doch ins Gesicht schlägt».

19 Gleichzeitig jedoch hielt er fest, dies setze voraus,

«dass wir selber gute Richter haben und dass deren absolute Unabhängigkeit durch Gesetz und rechtsverbindlichen Usus gewährleistet sein muss».

20 Dass dem seiner Auffassung nach jedoch nicht so war, ergab sich daraus, dass er die Führung von Strafverfahren und insbesondere deren Länge in der Schweiz harsch kritisierte $^{19}$.

21 Der Waadtländer Radikaldemokrat (FDP) Jean-Jacques Cevey erklärte, wir würden die EMRK mit dem Willen ratifizieren, es wesentlich besser zu machen als mehrere andere Unterzeichnerstaaten, im Respekt vor den Grundsätzen des Völkerrechts, die wir im Laufe unserer Geschichte unterzeichnet haben $^{20}$.

22 Als letzter Redner aus dem Plenum wandte sich Claude Bonnard (VD, Liberale Partei) an den Rat und unterstützte den Beitritt ebenso ${ }^{21}$.

23 Von Bundesrat Pierre Graber ist auch im Nationalrat kein einziges Wort der Beschwich-

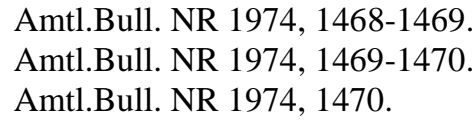

\footnotetext{
19 Amtl.Bull. NR 1974, 1471.

Amtl.Bull. NR 1974, 1472.

Amtl.Bull. NR 1974, 1472-1473.
} 
tigung ersichtlich; eine solche war angesichts dieser fast durchwegs positiven, ja begeisterten Voten aus dem Rate offensichtlich auch nicht notwendig.

Von den angeblich grossen Vorbehalten gegenüber der Ratifikation der EMRK im Parlament, von denen Paul Widmer zu wissen vorgibt, somit keine Spur. Im Gegenteil: weitestgehende Zustimmung und Erleichterung, dass der längst fällige Schritt endlich vollzogen werden kann, nachdem die bisher bedeutendsten Hindernisse, die einer Ratifizierung im Wege standen - fehlendes Frauenstimmrecht und konfessionelle Ausnahmeartikel der Bundesverfassung - hatten beseitigt werden können ${ }^{22,23}$.

Bundesrat Pierre Graber befasste sich in seinem Schlusswort vor allem mit Fragen im Zusammenhang mit einer eventuellen Unterstellung des Beschlusses des Parlamentes unter das fakultative Referendum - was er mit der Mehrheit der meisten Redner ablehnte. Von einem Bemühen, besorgte Volksvertreter $\mathrm{zu}$ beschwichtigen, oder gar von einem Satz, es sei unvorstellbar, dass die Schweiz mit ihren hohen Standards je wegen Verletzung von Menschenrechten verurteilt würde, berichtet das Amtliche Bulletin der Verhandlungen nirgends ${ }^{24}$. Wiederum ist das Gegenteil richtig: Es wurde mehrfach darauf hingewiesen, dass eine Verurteilung der Schweiz nicht etwa die Folge hätte, dass dadurch ein Urteil des Bundesgerichtes aufgehoben würde; damit bleibe es der Schweiz überlassen, wie sie auf ein solches Urteil reagiere, um das so festgestellte Unrecht dann dauerhaft zu beseitigen $^{25}$.

26 So muss denn schleierhaft bleiben, woher Paul Widmer seine diesbezügliche Weisheit bezogen hat ${ }^{26}$.

\section{Die «Bilanz» der Beschwerden gegen die Schweiz}

27 Was Paul Widmer zur sogenannten «Bilanz» der Beschwerden gegen die Schweiz ausführt, ist ebenfalls nicht zutreffend: Er behauptet, im letzten Jahrzehnt seien im Schnitt jährlich 250 Beschwerden eingereicht worden, von denen 90 Prozent vom Strassburger Gerichtshof als unbegründet abgewiesen worden seien ${ }^{27}$.

28 Betrachten wir zuerst die Statistik des EGMR für die Schweiz für die Jahre 1974 bis und mit 2008. In diesen rund 34 Jahren sind insgesamt 3'379 Beschwerden gegen die Schweiz eingereicht worden, von denen 3'295 als «offensichtlich unzulässig» ausschieden. Nur in zwei Prozent der Beschwerden fällte der Gerichtshof ein Urteil. Von 84 Urteilen kam es in $69 \% \mathrm{zu}$ einer Verurteilung der Schweiz. In $23 \%$ der Fälle wurde keine Verletzung der EMRK festgestellt. $6 \%$ der Fälle endeten mit
22 Am 7. Februar 1971 wurde die Vorlage zur Einführung des Frauenstimmrechts auf Bundesebene vom männlichen Stimmvolk mit 621'109 gegen 323'882 Stimmen (65,7 Prozent Ja) und von 151/2 Ständen gegen 61/2 Stände angenommen.

23 In der Volksabstimmung vom 20. Mai 1973 wurde der Bundesbeschluss über die Aufhebung des Jesuiten- und des Klosterartikels der Bundesverfassung angenommen und damit diese Artikel aus der Verfassung gestrichen. Das Verbot der Wahl von Geistlichen in den Nationalrat fiel jedoch erst mit dem Ersatz der Bundesverfassung von 1874 durch die sogenannte «Nachführung» der Bundesverfassung von 1999 dahin; es spielte im
Zusammenhang mit der EMRK keine Rolle.

24 Amtl.Bull. NR 1974,1502-1503.

25 So etwa Hefti, Amtl.Bull. SR 1974, 380.

26 Die Behauptung Widmers erinnert an die Anekdote über jenen Zürcher Anwalt, der nach einem feurigen Plädoyer den Prozess seines Klienten dennoch verlor, vom Gegenanwalt mit der Frage angesprochen worden war: «Aber Herr Kollega, wie konnten Sie nur Derartiges behaupten?», auf diese Anfrage den Zeigefinger der rechten Hand senkrecht nach oben streckte und bemerkte: «Herr Kollega, n'est-ce pas, le juge peut toujours se tromper!».

27 Siehe Fussnote 1. 
einer gütlichen Einigung. Die restlichen zwei Prozent der Urteile entfallen auf «Andere Entscheidungen ${ }^{28}$.

Was die «letzten Jahre» anbelangt, von denen Paul Widmer gesprochen hat, stellt sich die Lage so dar: Zwischen 2002 und 2013 gingen 3‘629 Beschwerden ein; im Durchschnitt somit etwa 363, wie sich den Angaben des Bundesrates in seiner Antwort vom 12. Februar 2014 auf die Interpellation Peter Keller entnehmen lässt ${ }^{29}$.

\section{Die Art der festgestellten Verletzungen der EMRK}

Verurteilungen erfolgten 1974-2008 in $32 \%$ der Fälle - also etwa einem Drittel - wegen Verletzung des Rechts auf ein faires Gerichtsverfahren (Art. 6 EMRK), in $18 \%$ wegen Verletzung von Art. 5 EMRK (Recht auf Freiheit und Sicherheit - also in Fragen des Freiheitsentzuges), nur in $17 \%$ wegen Verletzung des Anspruchs auf Achtung des Privat- und Familienlebens (Art. 8), in $15 \%$ wegen Verletzung des Rechts auf Äusserungsfreiheit (Art. 10), und der Rest von $18 \%$ entfiel auf andere Artikel der EMRK.

31 Die Behauptung Widmers, Verurteilungen wegen Verletzung des Rechts auf Privat- und Familienleben seien besonders häufig, fällt damit in sich zusammen: Nur gerade ein Sechstel der Verurteilungen entfällt auf diesen Bereich.

Dass Verurteilungen durch den EGMR in einem Staat zur Folge haben können, dass er möglicherweise ein Gesetz ändern muss, ge- hört zum gewöhnlichen Risiko, wenn man sich einer Menschenrechts-Gerichtsbarkeit unterstellt. Paul Widmer hat diesen Umstand in seiner Äusserung in der NZZ jedoch als «grosse Konsequenz» dargestellt, denn «der verurteilte Staat muss Vorkehrungen treffen, damit sich Fälle der beanstandeten Art nicht mehr ereignen».

33 Dies ist allerdings nicht mehr und nicht weniger als der Sinn einer Unterstellung unter die EMRK und deren Gerichtsbarkeit: Was als menschenrechtswidrige Regelung erkannt worden ist, soll für alle Zukunft korrigiert werden und sich wenn immer möglich nicht mehr wiederholen: Eine Haltung des «Wasch mir den Pelz, aber mach' mich nicht nass»», wie sie Widmer sichtlich vorschwebt, - sich vordergründig grossartig verpflichten, aber eigentlich nur zum Schein -, steht im Übrigen in absolutem Widerspruch zum grundlegenden Artikel 26 des Wiener Übereinkommens über das Recht der Verträge:

«Ist ein Vertrag in Kraft, so bindet er die Vertragsparteien und ist von ihnen nach Treu und Glauben zu erfüllen.»

\section{Unserem Rechtsempfinden widerstrebende Urteile?}

34 Paul Widmer wagt ausserdem eine rückwärtsgewandte Prognose, indem er behauptet: «Nicht selten widerstreben die Strassburger Urteile unserem Rechtsempfinden. Die eidgenössischen Kammern hätten die EMRK wohl nicht ratifiziert, wenn sie gewusst hätten, weshalb die Schweiz inskünftig verurteilt würde.» ${ }^{30}$
28 EGMR Statistics by country 1959-2008, (S. 131-133).

29 Interpellation Peter Keller (13.4174): Fremdes Recht vor Schweizer Recht?

30 Siehe Fussnote 1. Rückwärtsgewandte Prognosen sind noch schwieriger als jene, die sich auf die Zukunft beziehen (Mark Twain). Die letzteren lassen sich durch
Zeitablauf verifizieren oder falsifizieren; die Zeitmaschine, die es gestatten würde, dies auch für die Vergangenheit zu tun, ist leider oder zum Glück noch nicht erfunden worden.

31 Siehe FN 1. Das ist etwa so richtig, wie wenn behauptet würde, es sei besonders häufig, dass die Fussball-Na- 
Wessen Rechtsempfinden durch die Urteile aus Strassburg wirklich betroffen ist, lässt Widmer offen; das Pronomen «unserem» bleibt unbestimmt. Ist es das Rechtsempfinden der Mehrheit der Schweizerinnen und Schweizer, oder jener, von denen das Bonmot sagt, das Schönste am Regieren sei die Willkür?

Weshalb Widmer eine ausserordentliche Häufigkeit solcher Entscheidungen behauptet, bleibt schleierhaft ${ }^{31}$. Urteile aus Strassburg, welche mit Rücksicht auf die jeweiligen Familienverhältnisse im Einzelfall und die tatsächliche Bedeutung der einem Beschwerdeführer zur Last gelegten Gesetzesverstösse dazu führen, dass eine durch ein schweizerisches Gericht verfügte Ausweisung aus der Schweiz als Verstoss gegen die EMRK gewertet wird, eignen sich ganz besonders für populistisches Gepolter gegen «Strassburg».

Analysiert man die einzelnen Fälle jedoch unvoreingenommen, zeigt sich, dass der Menschenrechtsgerichtshof dem Zusammenleben bzw. der tatsächlichen Möglichkeit der Ausübung intensiver Beziehungen unter den Mitgliedern einer Familie in relativer Nähe zueinander und insbesondere im gleichen Land eine wesentlich höhere Bedeutung zumisst, als dies unsere Gerichte zu tun pflegen, und er gewichtet bei seiner Abwägung jeweils eine erhebliche Reihe von Kriterien, um zu seinen Entscheiden zu kommen.

Das hat wohl damit zu tun, dass in den meisten europäischen Staaten, welche zur EMRK ge- hören $^{32}$, sowohl Begriff als auch Faktizität der Familie mit ihrem intensiven Zusammenhalt zwischen deren Angehörigen wesentlich stärker ist als in der Schweiz, in welcher nicht zuletzt zufolge der hervorragenden wirtschaftlichen Situation der meisten ihrer Einwohnerinnen und Einwohner die Vereinzelung sehr weit entwickelt ist und sehr viel weniger auf gegenseitige Unterstützung innerhalb eines Familienverbandes zurückgegriffen werden muss $^{33}$.

\section{Zehn Fälle in Ausweisungssachen - nur drei Verurteilungen}

39 Untersucht man die Urteile des EGMR gegen die Schweiz, welche seit 2010 in Fällen ergangen sind, in welchen es sich um die Frage der Ausweisung von Ausländern aus der Schweiz gehandelt hat, findet man insgesamt zehn Entscheidungen:

- Gezginci (Türkischer Staatsangehöriger, Urteil vom 9. Dezember 2010)

- Shala (Staatsangehöriger des Kosovo, Urteil vom 15. November 2011)

- Kissiwa Koffi (Staatsangehörige der Elfenbeinküste, Urteil vom 15. November 2011)

- Udeh (Staatsangehöriger von Nigeria, Urteil vom 16. April 2013)

- Hasanbasic (Staatsangehöriger von Bosnien-Herzegowina, Urteil vom 11. Juni 2013)

- Berisha (Staatsangehöriger des Kosovo, Urteil vom 30. Juli 2013)
tional-Elf besonders häufig mehrere Tore hintereinander in 17 Minuten schiesse: Richtig ist bloss, dass in letzter Zeit mehrere Urteile sich mit solchen Fragen zu befassen hatten. Dies dürfte darauf zu rückzuführen sein, dass Schweizer Gerichte unter dem Eindruck populistischer Kampagnen bei der Beurteilung solcher Fragen vor allem die Interessen der mitbetroffenen $\mathrm{Fa}-$ milienmitglieder zu wenig gewichtet haben.

32 Es fehlen nur noch Weissrussland und der Vatikan. Letzterer hat wohl kaum je eine Chance, dem Europarat beizutreten, da er in keiner Art und Weise demokratisch organisiert ist, wogegen für Weissrussland ein Wechsel zur Demokratie noch immer erhofft werden darf.

33 Das Bundesamt für Statistik hat in seiner Untersuchung über «Privathaushalte nach Kanton und Haushaltstyp, 2012», festgestellt, dass in der Schweiz von insgesamt 3'553'711 Haushalten deren 1'261'750 auf Einpersonenhaushalte entfallen. Das sind 35,5\% aller Haushalte. 
- Vasquez (Staatsangehöriger von Peru, Urteil vom 26. November 2013)

- Palanci (Staatsangehöriger der Türkei, Urteil vom 25. März 2013)

- Ukaj (Staatsangehöriger des Kosovo, Urteil vom 26. April 2014)

- M.P.E.V. (Staatsangehörige von Ecuador, Urteil vom 8. Juli 2014).

Der Gerichtshof hat in sieben dieser Fälle keine Verletzung der EMRK durch die Schweiz gefunden. Hingegen ist er in drei Fällen - Udeh, Hasanbasic und M.P.E.V. - zu einer Verurteilung der Schweiz gelangt. Die Urteile Hasanbasic und M.P.E.V. sind einstimmig, also jeweils mit sieben Stimmen, ergangen $^{34}$; im Fall Udeh erfolgte die Verurteilung der Schweiz mit fünf gegen zwei Stimmen.

Im Laufe der Jahre 2010 bis 2014 waren in diesen zehn Schweizer Fällen insgesamt 22 Richter aus 18 europäischen Ländern tätig. Deren Mehrzahl, nämlich zwölf, stammte aus dem «westlichen» Teil Europas, also aus Ländern, die vor dem Beitritt der Schweiz zur EMRK bereits Vertragsstaaten waren (in Klammern an erster Stelle die Anzahl der Stimmen, die auf Nicht-Verletzung, also Abweisung der Beschwerde, an zweiter Stelle die Anzahl der Stimmen, die auf Verletzung, also Gutheissung der Beschwerde, von Richtern des jeweiligen Staates abgegeben worden sind): Belgien (3/1), Dänemark (5/2), Griechenland (1/0), Island (1/0), Italien (5/4), Luxemburg (0/1), Norwegen (0/1), Österreich (1/0), Portugal (0/4), der Schweiz (7/3), Türkei (1/4) und Zypern (1/0). Die übrigen Richter stammten aus Kroatien (1/0), Litauen (5/3), Monaco (1/0), Montenegro (3/3), Serbien(2/2)

34 Sie würden somit sogar die Anforderungen erfüllen, die Paul Widmer in seinem Artikel als wünschenswert angeführt hat, nämlich dass Verurteilungen von Staaten durch den EGMR nur noch einstimmig oder wenigstens und Ungarn(4/3). Unterscheidet mannachStaaten, die dem früheren «Westen» oder «Osten» angehört haben, ergibt sich für die westlichen Staaten ein Verhältnis von 27 zu 17 (38 \% für Verurteilung), für jene des Ostens ein solches von $15 \mathrm{zu} 11$ (42\% für Verurteilung). Man sieht: Die Differenz zwischen Ost und West ist mit nur gerade $4 \%$ ganz offensichtlich nicht signifikant und keineswegs entscheidend.

\section{Vorwürfe fallen in sich zusammen}

42 Die Analyse dieser Fälle zeigt somit eines ganz deutlich: Offensichtlich stört in erster Linie das nicht einstimmig ergangene Urteil Udeh die Kritiker des EGMR, die es jedoch versäumt haben, diesem die sieben anderen abweisenden - Urteile gegenüberzustellen. Sie stellen somit die Rechtsprechung des EGMR in verzerrter Weise dar.

43 Dies ist wohl darauf zurückzuführen, dass die Medien im Allgemeinen und die dem EGMR aus ihrer parteiideologischen Einstellung heraus feindlich gesinnten Blätter im Besonderen kaum je über jene Urteile breit berichten, in welchen Beschwerden gegen die Schweiz abgewiesen wurden. Hervorgehoben werden dagegen Urteile, die zu einer Verurteilung der Schweiz geführt haben. Das ist Ausfluss des die Medienwelt beherrschenden Prinzips «Hund beisst Mann ist keine Nachricht, Mann beisst Hund ist eine Nachricht». Daraus ist für Politik und Wissenschaft der Schluss zu ziehen: Nachrichten aus Zeitungen mit grossen Buchstaben, die Empörung zu bewirtschaften versuchen (und jene, die es ihnen auch ohne grosse Buchstaben gleichzutun versuchen), werden in ihrer tatsächlichen Bedeutung zumeist stark überschätzt ${ }^{35}$.

mit sechs von sieben Stimmen erfolgen sollten.

35 Ganz abgesehen davon, dass seit mehr als hundert Jahren sich die Frage der Qualifikation derjenigen Personen, die in Medien tätig sind, andauernd in gleicher 


\section{Einzelfälle oder Grundsatzfragen?}

44 In seiner Kritik des EGMR erwähnt Paul Widmer sodann noch fünf ganz andere Fälle von Verurteilungen durch eine Kammer des EGMR, welche die Schweiz betroffen haben, so dass anzunehmen ist, er betrachte auch diese Verurteilungen als «Grenzüberschreitungen» seitens der Strassburger Richter, von denen er mehr «richterliche Zurückhaltung» einfordert. Angesprochen sind die Fälle Gross $^{36}$, Schlumpf ${ }^{37}$, Verein Rhino ${ }^{38}$, Perinçek $^{39}$ und Glor ${ }^{40}$.

In formeller Hinsicht ist festzuhalten, dass die Urteile Gross und Perinçek noch gar nicht rechtskräftig sind, da Anträge der Schweiz, diese Fälle von der Grossen Kammer überprüfen zu lassen, erfolgreich waren ${ }^{41}$.

46 Sie betreffen somit nach Auffassung des Strassburger Filterkomitees wichtige Grundsatzfragen.

\section{Der Anspruch auf einen sicheren Suizid auch ohne Krankheit}

47 Im Fall Gross hat die Kammer des EGMR richtigerweise festgestellt, dass das Schweizer Recht es seit dem Entscheid BGE 133 I 58 im Unklaren lässt, ob ein Arzt berechtigt ist, einer Person, die nicht im eigentlichen Sinne als
Weise stellt: Inwieweit sind Journalisten Personen, die in der Lage sind, das, worüber sie berichten, so darzustellen, dass es sowohl im Inhalt als auch im Gewicht der Wahrheit entspricht? Schon 1906 schrieb George Bernard Shaw in einer Regieanweisung in seinem Stück «The Doctor's Dilemma»: «Walpole kehrt mit dem Reporter (Original: The Newspaper Man) zurück, einem heiteren freundlichen jungen Mann, der für die gewöhnlichen Geschäfte infolge eines angeborenen geistigen Gebrechens untauglich ist: er ist nämlich unfähig, das, was er sieht, genau zu beschreiben, oder das, was er hört, genau zu verstehen oder zu erzählen. Da die einzige Beschäftigung, bei der diese Mängel nicht schaden, der Journalismus ist - eine Zeitung braucht ja nicht gemäss ihren Beschreibungen und Berichten zu handeln, sondern sie bloss an neugierige Faulpelze zu verkaufen, verliert also durch Ungenauigkeit und Unwahrhaftigkeit nur ihre Ehre -, so musste er unbedingt durch eine force majeure Journalist werden und trachten, trotz eines täglichen Kampfes mit seinem Mangel an Bildung und seiner prekären Beschäftigung, stets guten Mutes zu erscheinen. Er hat ein Notizbuch bei sich und versucht gelegentlich eine Notiz zu machen, da er aber nicht stenographieren und überhaupt nicht schnell schreiben kann, gibt er das, ehe er einen Satz zustande gebracht hat, gewöhnlich als verlorene Mühe auf.»

36 Urteil des EGMR 67810/10 vom 14. Mai 2013 (Gross gegen Schweiz); vom Ausschuss der Grossen Kammer am 7. Oktober 2013 zur Neubeurteilung angenommen; nzz.ch vom 8. Oktober 2013 (EGMR entscheidet über Schweizer Suizidhilfe-Fall).

37 Urteil des EGMR 29002/06 vom 8. Januar 2009 (Schlumpf gegen Schweiz).

38 Urteil des EGMR 48848/07 vom 11. Oktober 2011 (Association Rhino und andere gegen Schweiz).
39 Urteil des EGMR 27510/08 vom 17. Dezember 2013 (Perinçek gegen Schweiz); vom Ausschuss der Grossen Kammer am 2. Juni 2014 zur Neubeurteilung angenommen; nzz.ch vom 3. Juni 2014 (Völkermord-Urteil wird überprüft).

40 Urteil des EGMR 13444/04 vom 30. April 2009 (Glor gegen Schweiz).

41 Im Fall Gross hat sich gar eine Situation ergeben, die nach Kenntnis des Autors bisher einmalig ist: Nachdem der Fall an die Grosse Kammer überwiesen worden ist, stellten die Bundesbehörden fest, dass die Beschwerdeführerin gar nicht mehr lebt. Gegenüber der Grossen Kammer beschuldigten sie deren Rechtsvertreter, dies arglistig verschwiegen zu haben. Dieser konnte jedoch nachweisen, dass die Beschwerdeführerin durch einen Pfarrer im Auftrag von Exit (Deutsche Schweiz) begleitet worden ist, welcher ihr unter dem Siegel des Berufsgeheimnisses eines Geistlichen versichern musste, niemandem davon etwas zu sagen. Da weder die EMRK noch die Verfahrensordnung des Gerichtshofes sich darüber äussern, was mit einer Beschwerde geschieht, deren Urheber nicht mehr lebt, ist das Schicksal dieser Beschwerde zum gegenwärtigen Zeitpunkt offen. «Strassburg» hat im Fall Tyrer gegen das Vereinigte Königreich dessen Rückzug der Beschwerde (die sich gegen die Anwendung der Prügelstrafe auf der Isle of Man richtete) nicht zum Anlass genommen, das Verfahren einzustellen. Es führte den Fall wegen der grundsätzlichen Bedeutung ohne Beteiligung des Beschwerdeführers weiter und verurteilte in der Folge Grossbritannien. Dies lässt darauf schliessen, dass in Strassburger Fällen der Gerichtshof und nicht der Beschwerdeführer Herr des Verfahrens ist. In der Folge erneuerte die Londoner Regierung während zwölf Jahren die Unterstellung des Gebietes der Isle of Man unter die Gerichtsbarkeit des EGMR nicht mehr. 
krank gilt, ein Rezept für eine letale Dosis Natrium-Pentobarbital (NaP) auszustellen. In seinem Entscheid hat das Bundesgericht in Erwägung 6 klar gesagt, es gehöre zum Selbstbestimmungsrecht jedes Menschen, selber zu entscheiden, wann und wie er sterben wolle. Damit ist erstmals das Recht oder die Freiheit zum Suizid ausdrücklich als Menschenrecht anerkannt worden, und der EGMR hat diese Betrachtungsweise in der Folge übernommen $^{42}$.

Da amerikanische Forschungsergebnisse gemäss Bundesrat (in seiner Antwort vom 9. Januar 2002 auf die einfache Anfrage von Andreas Gross zu Suiziden und Suizidversuchen $^{43}$ ) nahelegten, dass bis zu 49 von $50 \mathrm{Su}$ izidversuchen scheitern, erscheint ein einsamer Suizidversuch einer Person nicht als ausreichende Möglichkeit, um von diesem Recht Gebrauch zu machen: Wer mit einem Risiko von 49 zu 1 damit rechnen muss, mit einem Suizidversuch zu scheitern, kann das durch die EMRK verbürgte Recht nicht effizient und praktisch in Anspruch nehmen. Aus der Strassburger Artico-Rechtsprechung ${ }^{44}$ ergibt sich jedoch ein Anspruch gegenüber dem Staat, ein solches Recht mindestens mit an Sicherheit grenzender Wahrscheinlichkeit in Anspruch nehmen zu können. Dies macht es unausweichlich, dass jemand über die Möglichkeit verfügen können muss, zur Durchführung eines Suizids ${ }^{45}$ sachkundige Hilfe in Anspruch zu nehmen, wozu auch gehört, dass da- für das beste Suizidmittel zur Verfügung stehen muss.

49 Somit steht die gegenwärtige Rechtsunsicherheit in Bezug auf die Verschreibung von $\mathrm{NaP}$ für Personen, die nicht krank sind, im Widerspruch zur EMRK. Im Fall Gross ging es darum, diesen Widerspruch aufzulösen. Dies ist angesichts der grossen Unterstützung weiter Teile der Bevölkerung für die Möglichkeit eines Suizids bei Lebenssattheit im Alter ${ }^{46}$ eine bedeutsame Grundsatzfrage, die eben deshalb, weil das Bundesgericht bei deren Beantwortung offensichtlich versagt hat, nun durch den EGMR zu entscheiden ist: Muss ein Staat, der nichts dagegen einzuwenden hat, dass Ärzte kranken Personen NaP zum Zwecke eines begleiteten Suizids verschreiben, dafür sorgen, dass nicht Kranke, die im Einzelfall auch einen guten Grund dafür haben können, ihr Leben selbst vorzeitig zu beenden, jenen gegenüber nicht diskriminiert werden dürfen?

50 Eine der innerstaatlich denkbaren Lösungen dieses Problems wäre, dass zur medizinischen Indikation hinzu dem Arzt auch eine juristische Indikation als Grundlage für eine Rezeptierung zur Verfügung gestellt wird für den Fall, dass er einen beabsichtigten begleiteten Suizid für gerechtfertigt hält ${ }^{47}$.
42 Im Urteil des EGMR 31322/07 vom 20. Januar 2011 (Haas gegen Schweiz), Rn. 51.

43 Einfache Anfrage Andreas Gross (01.1105): Suizide und Suizidversuche. Zahlen.

44 Im Urteil des EGMR 6694/74 vom 13. Mai 1980 (Artico gegen Italien) hat der Gerichtshof in Abschnitt 33 festgehalten, «The Court recalls that the Convention is intended to guarantee not rights that are theoretical or illusory but rights that are practical and effective . . .» und aus dieser Überlegung positive Pflichten für Staaten abgeleitet, wenn der konkrete Rechtszustand zur
Folge hätte, dass Rechte und Freiheiten bloss theoretisch oder gar illusorisch bleiben.

45 Gemeint ist dabei selbstverständlich stets ein Suizid, der bei vernünftiger Betrachtung als gerechtfertigt erscheinen kann.

46 Siehe beispielsweise die Berichterstattung $\mathrm{zu}$ diesem Thema in der Mitgliederzeitschrift von EXIT (Deutsche Schweiz) Nr. 2/2014.

47 Als Beispiel für einen gangbaren Lösungsvorschlag sei auf meinen Beitrag in der NZZ vom 21. Mai 2013, S. 18, verwiesen (Sterbehilfe - neue Gesetze nicht nötig). 
7. Die grosse Bedeutung der Äusserungsfreiheit

51 Im Fall Perinçek geht es - nach dem Recht auf Leben - um das m. E. zweitwichtigste Menschenrecht: Das Recht, sich ungehindert äussern zu dürfen. Mit ihm allein lassen sich alle anderen Menschenrechte erkämpfen, denn nur wer sich frei äussern kann, kann auch Andere dazu bewegen, sich einer Idee anzuschliessen und sie eines Tages mehrheitsfähig zu machen.

Der türkische Politiker Perinçek wurde in der Schweiz verurteilt, weil er den Genozid an den Armeniern durch die osmanische Türkei hier öffentlich geleugnet hat. Nach schweizerischer Ansicht hat er dadurch die RassismusStrafnorm (Art. 261 ${ }^{\text {bis }}$ StGB) verletzt. Die Kammer, welche diese Frage zu beurteilen hatte, fand, der Begriff des Genozids oder Völkermords sei nicht eindeutig festgelegt. Seine Anwendung auf die damalige türkische «Lösung» der Armenierfrage sei kontrovers. Deshalb hat sie die Verurteilung Perinçeks durch die schweizerischen Gerichte als Verletzung der Äusserungsfreiheit qualifiziert.

Man wird nicht sagen können, diese Betrachtungsweise des EGMR sei von vornherein falsch oder irrig, sondern wird anerkennen müssen, dass angesichts der konkreten Umstände der Diskussion dieser historischen Frage dieser Standpunkt durchaus auch eingenommen werden kann. Somit sind Vorwürfe gegenüber der urteilenden Kammer nicht am Platz. Sollte die Grosse Kammer den Fall anders sehen - was durchaus offen ist -, kann dannzumal $^{48}$ das neue Urteil wieder diskutiert werden.
54 Es liegt eben in der Natur der Sache, dass überall dort, wo Rechtssachen einem Rechtszug von einer Instanz zur nächsten unterliegen, auf den verschiedenen Ebenen auch unterschiedliche Wertungen in den Urteilen möglich sind. Dies ist aber nie ein Grund dafür, die Abschaffung solcher Justizgremien zu fordern oder gar - wie hier - sich aus dem Kreis der einigermassen zivilisierten Staaten entfernen zu wollen.

\section{Vereinigungsfreiheit als wichtiges politisches Recht}

55 Eine gewisse Ähnlichkeit mit dem Fall Perinçek weist auch der Fall des in Genf beheimateten Vereins Rhino auf, der gemäss Vereinszweck das Ziel verfolgte, leere Liegenschaften durch Besetzung einer Zwischennutzung zuzuführen, um den besonders in Genf spürbaren erheblichen Mangel an Wohnraum zu lindern. In jenem Fall hatten die Gerichte in der Schweiz diesen Vereinszweck als rechtswidrig eingestuft und gestützt darauf den Verein von Amtes wegen auflösen wollen. Der EGMR hat dies als Verletzung von Art. 11 der EMRK - Vereinigungsfreiheit - qualifiziert, und zwar einzig und allein deshalb, weil es der Schweiz im Strassburger Verfahren nicht gelungen ist, darzutun, dass es kein milderes Mittel gegeben hat, um das Verhalten des Vereins in rechtlich allenfalls noch zu duldende Schranken zu weisen.

56 Auch hier spürt man bei der Lektüre des Urteils, dass den Strassburger Richtern die Garantien der EMRK eben wirklich wichtig sind, und dass sie bei der Frage, ob der angefochtene Eingriff in einer demokratischen Gesellschaft notwendig war, richtigerweise verhältnismässig strenge Massstäbe anlegen. Das Recht auf Vereinigung darf wohl als das drittwichtigste Menschenrecht angesehen

in Strassburg stattfinden. 
werden, weil es gemeinsam mit der Äusserungsfreiheit den Kampf einzelner Gruppen um Ausweitung der Freiheitsbereiche ermöglicht.

Die entsprechende Verurteilung ist die Konsequenz aus der mangelnden Bemühung einheimischer Richter, die richtige Balance zwischen Freiheit und Ordnung zu finden. Die Strassburger Rechtsprechung sollte somit als Chance begriffen werden, im eigenen Land das Nachdenken über diese wichtigen Garantien bei den Gerichten zu fördern und den Grundrechten auch in der Praxis die Bedeutung zuzuerkennen, die ihnen zukommt.

\section{Heikle Abgrenzungen im Recht können zu Diskriminierung führen}

58 Die beiden übrigen Urteile, die Paul Widmer aufgestossen sind, weisen bei aller Verschiedenheit ihrer materiellen Hintergründe Pflicht zur Zahlung von Militärpflichtersatz bei Untauglichkeit zur Leistung von Militärdienst im Fall Glor, Verpflichtung der Krankenkasse zur Tragung von Kosten einer geschlechtsangleichenden Operation im Fall Schlumpf - einen gemeinsamen Nenner auf. In beiden Fällen sieht das inländische Gesetz eine Differenzierung zwischen analogen Situationen vor: Im Fall des Militärpflichtersatzes hat bisher nur eine schwere Dienstuntauglichkeit zur Befreiung von dessen Bezahlung geführt; im Fall der operativen Geschlechtsan- gleichung sieht das Bundesgericht unter anderem mindestens zwei Jahre psychiatrische Begleitung vor, bevor die Krankenkasse verpflichtet ist, die Kosten zu übernehmen.

59 Derartige Unterscheidungen können leicht zu Verletzungen von Garantien der EMRK führen, insbesondere Diskriminierungen, und diese sind in Verbindung mit sämtlichen EMRK-Rechten durch Art. 14 der Konvention verboten $^{49}$. Der Gesetzgeber täte somit gut daran, beim Erlass solcher Bestimmungen einen Moment innezuhalten und sich zu fragen, ob die dadurch geschaffene Diskriminierung vor Art. 14 der EMRK Bestand haben könnte.

60 Im Fall des an Diabetes leicht erkrankten Beschwerdeführers, für den in der Armee ohne weiteres ausserhalb kombattanter Truppenteile eine erfüllbare Aufgabe zu finden wäre (insbesondere in einer Armee, die seit 1847 keinen Krieg mehr hat führen müssen), erreichte diese Unterscheidung den Grad der Diskriminierung. Dazu darf festgestellt werden, dass früher das nationale Recht selbst bei schwerer Dienstuntauglichkeit Militärpflichtersatz vorsah. Die Folge der Strassburger Entscheidung - Verzicht auf die Pflicht zur Leistung von Militärpflichtersatz auch bei leichter Untauglichkeit - beseitigt somit lediglich eine Ungerechtigkeit, welche die Leitung der Armee bzw. die dafür verantwortlichen politischen Instanzen nach Meinung des Autors damals schon längst von sich aus hätten abschaffen können und müssen ${ }^{50}$.

Vertrag, welcher Menschenrechte und Grundfreiheiten schützt, macht es erforderlich, auch bei den Behörden ein entsprechendes Sensorium für Konfliktmöglichkeiten $\mathrm{zu}$ entwickeln und vorausschauend zu prüfen, wie durch entsprechende Änderung der Gesetzgebung das Risiko einer Verletzung vermindert werden kann. Dazu ist vielleicht auch von Interesse, dass der Bundesrat vor dem Beitritt der Schweiz zur EMRK Botschafter Emanuel Diez beauftragt hatte, das Bundesrecht und die 
Dies ist im Bereich des Militärrechts übrigens nichts Neues. In der ersten Beschwerde, die seinerzeit von der damals noch existierenden Europäischen Menschenrechtskommission gegen die Schweiz zugelassen worden war, beschwerte sich Herbert Eggs ${ }^{51}$ darüber, dass ihm wegen unbotmässigen Haarschnitts in der Armee die Strafe scharfen Arrests auferlegt worden war, ohne dass darüber je ein Richter hätte entscheiden können. Der Fall führte schliesslich zu einer Änderung der Militärdisziplinargerichtsordnung und letztlich zur Abschaffung der damaligen Haarschnittvorschrift: Heutzutage ist die Frisur eines Soldaten tabu; er hat lediglich dort, wo der Dienst dies erfordert, ein Haarnetz zu tragen. So hat die EMRK in ganz erfreulicher Weise dazu geführt, auch alte Militärzöpfe abzuschneiden und somit für eine diesbezügliche Haarschnittvorschrift in umgekehrter Richtung gesorgt.

Im Fall der Kosten der operativen Geschlechtsangleichung lagen die Verhältnisse so, dass die Beschwerdeführerin bereits etwas fortgeschrittenen Alters war, und dass demzufolge der Sinn der vom Bundesgericht aufgestellten Bestimmung, welche die sakrosankte Zweijahresfrist als Voraussetzung für das Stellen einer gesicherten Diagnose vorsah, keinerlei Berechtigung mehr gehabt hat.

kantonalen Rechte auf denkbare Konfliktpotentiale zu untersuchen. Es wird berichtet, Diez sei nach kurzer Zeit an den Bundesrat gelangt und habe mitgeteilt, die Aufgabe sei nicht zu bewältigen; man müsse die Risiken in Kauf nehmen (seinerzeitige persönliche Mitteilung von alt Nationalrat Ernst Bieri (FDP, ZH) an den Verfasser).

51 European Commission of Human Rights, Decisions and Reports (DR), Band 6, S. 170-181 (Zulässigkeitsentscheidung vom 11. Dezember 1976); Band 15, S. 35-69. Die Menschenrechtskommission schloss mit zwölf gegen zwei Stimmen auf eine Verletzung von Art. 5 Abs. 1 EMRK. Da damals weder die Schweiz noch die Kommission den Gerichtshof anriefen, hatte das Ministerkomitee des Europarates definitiv zu entscheiden. Da es der erste Fall gegen die Schweiz war, und nachdem der Bundesrat $\mathrm{zu}$ verstehen gegeben

\section{Fazit}

63 Die vorstehenden Ausführungen zeigen, dass der von Paul Widmer (und anderen) geäusserten Kritik an der Rechtsprechung des EGMR bezüglich der Schweizer Fälle keinerlei tragfähige Tatsachen zugrunde liegen. Diese Rechtsprechung weiss auch in den heiklen Ausweisungsfällen sehr wohl zu differenzieren; ein präponderanter Einfluss von Richtern, deren Familiennamen auf osteuropäische Endsilben enden, ist nicht zu beweisen; andere oft angeführte angebliche Einzelfälle erweisen sich durchwegs als Kristallisationspunkte wichtiger Grundsatzfragen. Somit kann festgestellt werden, dass die Kritik, wie sie Widmer und andere an der Spruchpraxis des EGMR gegenüber der Schweiz geäussert haben, einer wissenschaftlichen Überprüfung nicht stand hält.

64 Die Schweiz hat deshalb allen Anlass, ihre Verbundenheit mit der Konvention zu betonen; sie allein, in Gemeinschaft mit der Tätigkeit des EGMR, sorgt dafür, dass Bürgerinnen und Bürger ein klares Unterscheidungsmerkmal zwischen zivilisierten und unzivilisierten Staaten zur Verfügung haben: Wer sich als europäischer Staat der EMRK unterstellt, sich bemüht, Rechte und Freiheiten der Menschen $\mathrm{zu}$ achten und den

hatte, dass er das Gesetz ändern wolle, traf das Ministerkomitee jedoch keine Entscheidung; weder für eine Verurteilung noch für eine Abweisung der Beschwerde erzielte es eine damals noch notwendige Zweidrittelsmehrheit. Erst nachdem das Ministerkomitee darauf folgende Schweizer Beschwerden, ebenfalls wegen Militärhaarschnitts, zu entscheiden hatte (Santschi und andere, DR 31, S. 5-49) stellte das Ministerkomitee fest, die Schweiz habe Art. 5 EMRK verletzt und sprach den dortigen Beschwerdeführern Entschädigungen zu. Herbert Eggs erkämpfte sich im Anschluss daran mittels einer neuen Beschwerde und im Rahmen einer dann vom nachmaligen Präsidenten der Kommission, Stefan Trechsel, vermittelten Gütlichen Einigung eine analoge Entschädigung, obwohl es an einem verurteilenden Erkenntnis gefehlt hatte. 
Entscheidungen des EGMR auch dort ohne Widerspruch nachzukommen, wo sie Teile der staatlichen Verwaltung und gewisse Mächtige schmerzen, darf als zivilisiert gelten, denn Zivilisation bedeutet Ablösung einer Herrschaft der Macht durch die Herrschaft des Rechts - wie seinerzeit beim Gegensatz zwischen den demokratisch gesinnten Griechen und den tyrannisch beherrschten Persern.

Dass der EGMR die Rechte und Freiheiten, welche die Konvention garantiert, dynamisch auslegt, entspringt nicht etwa seiner Willkür, wie gelegentlich insinuiert wird, sondern ist Auftrag und Gebot der die EMRK einleitenden Präambel. Sie verlangt im Interesse der «Herbeiführung einer grösseren Einigkeit unter» den Mitgliedern des Europarates und als «eines der Mittel zur Erreichung dieses Zieles», welches «in der Wahrung und in der Entwicklung der Menschenrechte und Grundfreiheiten besteht», dafür zu sorgen, dass die EMRK nicht dem sonst oft üblichen Schicksal von Gesetzen unterliegt, welches der Jurist und Rechtsanwalt Johann Wolfgang Goethe im Jahre 1808 so trefflich dargestellt hat ${ }^{52}$ : «Es erben sich Gesetz' und Rechte / wie eine ew'ge Krankheit fort; / Sie schleppen von Geschlecht sich zum Geschlechte, / Und rücken sacht von Ort zu Ort. / Vernunft wird Unsinn / Wohlthat Plage; / Weh dir, dass du ein Enkel bist! / Vom Rechte, das mit uns geboren ist / Von dem ist, leider! nie die Frage.»
66 Seit dem 28. November 1974 jedoch, dem Tage des Beitritts der Schweiz zur EMRK, ist dies auch für uns in der Schweiz ${ }^{53}$ - entgegen der Meinung Goethes - durchaus die Frage.

67 Demzufolge hat die Schweiz allen Anlass, ihr vierzigjähriges Dazugehören am 28. November 2014 mit Stolz zu begehen und sich ganz entschieden in die Gruppe der Verteidiger der EMRK einzureihen. Die vor uns stehenden Jahre mit ihren unguten Entwicklungen im europäischen Osten fordern uns einmal mehr im Bemühen, einen Rückfall in Barbarei zu vermeiden $^{54}$.
52 Faust. Erster Theil, Goethes Werke Herausgegeben im Auftrage der Grossherzogin Sophie von Sachsen, I. Abtheilung, 14. Band, Weimar 1887; Nachdruck DTV 1987; Zeilen 1972-1979; erstmals veröffentlicht 1808.

53 Die Schweiz ist vor wenigen Wochen im britischen Oberhaus im Rahmen einer Debatte um einen von Lord Falconer eingereichten Gesetzesentwurf zur Ermöglichung von Suizidbeihilfe von Lord Joffe als «arguably the most conservative country in Europe» («das wohl konservativste Land in Europa») bezeichnet worden. Siehe Hansard, House of Lords, 18 July 2014: Column 789.
54 Geschichtliche Fälle solcher Barbarei sind etwa die Haltung der Schweiz gegenüber fliehenden deutschen Juden, die an der Grenze abgewiesen worden sind, oder die Fälle jener administrativ Versorgter und Verdingkinder in der zweiten Hälfte des 20. Jahrhunderts; beides schlimme politische Auswüchse, die erst Jahrzehnte später als das erkannt worden sind, was sie waren. Die EMRK kann uns dank der Rechtsprechung des EGMR vor solchen Entgleisungen in der Gegenwart bewahren. 